\title{
ARTÍCULO ORIGINAL: Epidemiología de las infecciones pos-trasplante hepático en pacientes adultos operados en los Hospitales México y Calderón Guardia, durante el periodo del año 2000 a marzo del 2012
}

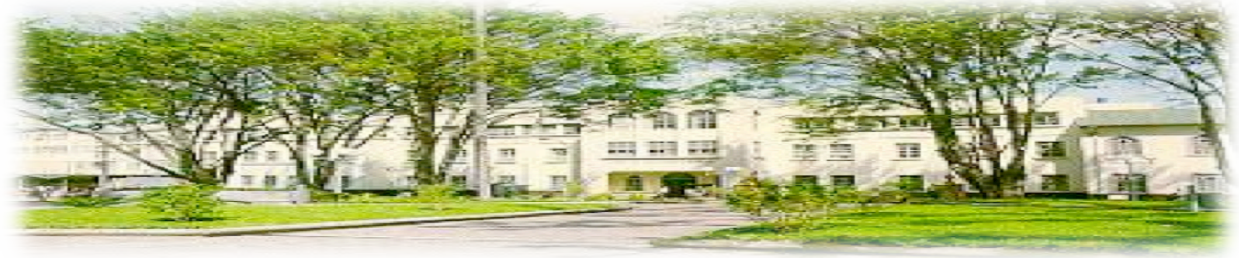

Haspital San quan de Dias. San José, Costa Rica. Fundada en 1845

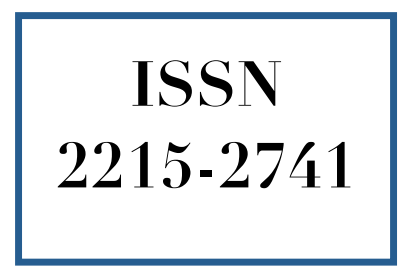

Recibido: $\quad 19 / 01 / 2014$

Aceptado: $\quad 21 / 04 / 2014$

A. Segura Irías ${ }^{1}$

M. Madrigal Borloz ${ }^{1}$

${ }^{\mathbf{1}}$ Programa Nacional de Trasplante Hepático. C.C.S.S. San José. Costa Rica.

\section{RESUMEN}

En los últimos años tanto en el Hospital México como en el Hospital Dr. Rafael Calderón Guardia se ha seguido la tendencia a nivel mundial respecto a un aislamiento de bacterias con mayor resistencia a antimicrobianos y hongos patógenos oportunistas en el paciente que sufre una infección. Al estar la población con trasplante hepático obligada a utilizar inmunosupresores, se convierte en un grupo susceptible a los microorganismos patógenos, en relación con un mayor riesgo de morbilidad y mortalidad intrahospitalaria. No se conocía la epidemiología, el comportamiento a través del tiempo, las complicaciones y la mortalidad por infecciones en los centros mencionados. Las infecciones nosocomiales más frecuentes en el primer mes posterior al trasplante hepático en la población estudiada estuvo relacionada con los procesos infecciosos que comprometieron el tracto respiratorio inferior.
El microrganismo que se aisló con mayor frecuencia fue Klebsiella pneumoniae productora de metalo beta-lactamasas en un $13 \%$ respecto al total de microorganismos aislados. El género, la edad o el diagnóstico previo de diabetes no fueron factores de riesgo con significancia estadística para desarrollar una infección. La fiebre en un $31.6 \%$ de los pacientes fue el síntoma que se presentó de forma más frecuente, concomitante al diagnóstico de infección. La mortalidad asociada a infección posterior a trasplante hepático fue de $21.1 \%$. Ni el uso de inmunosupresión ni la antibioticoterapia de forma empírica incrementó el riesgo de fallecer por una infección

\section{PALABRAS CLAVE}

Trasplante. Obtención de órganos y tejidos. Cuidados intensivos. 


\section{ABSTRACT}

In recent years, both the Mexico and in the Dr. Rafael Calderon Guardia Hospitals have followed the global trend regarding isolation of bacteria with increased resistance to antimicrobials and opportunistic fungal pathogens in patients suffering from infection. Transplant population are being forced to use immunosuppressive medication, so this population are a group susceptible to pathogens related to an increased risk of morbidity and hospital mortality. The most common nosocomial infections, in the first month after liver transplantation in the study population were related to infectious processes that compromised the lower respiratory tract. The microorganism most frequently isolated was Klebsiella pneumoniae metallo beta-lactamase producing by $13 \%$. Gender, age or previous diagnosis of diabetes were not significant statistically risk factors for developing an infection. Fever at $31.6 \%$ was the symptom that was presented more frequently, concomitant with the diagnosis of infection. The mortality associated with infection after liver transplantation was $21.1 \%$, neither the use of immunosuppression nor the use of an empirical antibiotic therapy increased the risk of death secondary to infection.

\section{KEY WORDS}

Transplantation. Tissue and organ procure. Intensive care.

\section{INTRODUCCIÓN}

Las dos principales causas que llevan a un trasplante hepático nivel mundial son el fallo hepático fulminante y la cirrosis hepática ${ }^{(1)}$. La prevalencia de estas patologías se mantiene en constante crecimiento, incluso en Costa Rica, mas la sobrevida ha cambiado de forma dramática tras la era post trasplante hepático ${ }^{(2)}$.

En la era pre-trasplante el fallo hepático fulminante era de pronóstico fatal, a nivel universal, con mortalidad que rondaba de un $80 \%$ a $90 \%$; de forma similar la cirrosis hepática descompensada mostraba mortalidad cercana a $50 \%$ a un año ${ }^{(3)}$. Para aquellos pacientes que llegan a presentar enfermedad hepática crónica en estadio terminal o fallo hepático fulminante, el trasplante hepático se ha convertido en uno de los métodos de terapia de elección, ya que les permite alcanzar una mejoría en la sobrevida y calidad de vida; tras la falta de métodos de sustitución hepática artificiales alternativos que hayan demostrado beneficio a largo plazo, y sólo contar con medios inmediatos de soporte previo a la realización del trasplante ${ }^{(4)}$.

Dentro de las principales complicaciones que puede presentar el paciente trasplantado hepático están las infecciones, siendo ellas por sí mismas un factor de riesgo para la disfunción del órgano $\operatorname{trasplantado}^{(5)}$. Por lo tanto uno de los principales factores de riesgo para la aparición de infecciones en estos pacientes es la terapia inmunosupresora.

Más del 90\% de las infecciones que ocurren en el primer mes son las mismas infecciones bacterianas y fúngicas relacionadas a tejidos blandos, pulmón, tracto urinario y accesos vasculares, que ocurren en la población de pacientes intervenidos quirúrgicamente sin inmunosupresión ${ }^{(6)}$.

Luego de haber sobrepasado el primer mes empiezan a emerger las infecciones por virus inmunorreguladores (CMV, EBV, virus Herpes, HBV, $\mathrm{HCV}, \mathrm{HIV})$. El estado de inmusupresión aunado a la infección con los virus mencionados aumenta la susceptibilidad a microorganismos oportunistas como Pneumocystis jiroveci, Aspergillus sp. y Listeria monocytogenes ${ }^{(6)}$.

Seis meses después del trasplante los pacientes pueden ser divididos en tres categorías. El 80\% de los trasplantados mantienen buena condición y sus cuadros infecciosos van a ser similares al de la población general, siendo las infecciones del tracto respiratorio las más frecuentes; la presencia de microorganismos oportunistas va a ser rara, excepto que haya una exposición prolongada al agente infeccioso ${ }^{(6)}$. Un $10 \%$ presenta infecciones crónicas y progresivas por $\mathrm{HBV}, \mathrm{HCV}$, CMV, EBV y papiloma virus y un $5 \%$ a $10 \%$ desarrollan rechazo crónico del órgano trasplantado que los lleva a utilizar altas dosis de inmunosupresores y los pone en un riesgo mayor de desarrollar infecciones virales crónicas y por lo tanto los vuelve más susceptibles a organismos oportunistas como Pneumocystis jiroveci, Listeria monocytogenes, $N$. asteroides, Cryptococcus neoformans y Aspergillus sp. ${ }^{(6)}$.

En Costa Rica, lamentablemente no se tiene estudios que abarquen la población en general y 
que indiquen con claridad la prevalencia de Enfermedad Hepática Crónica ni el Fallo Hepático Agudo, ni su distribución en cuanto a estadios.

Para abril de 2011, la cantidad total de trasplantes hepáticos en adultos y niños realizados en Costa Rica fue de 100. Sin embargo, se desconoce el comportamiento de las complicaciones en esta población, en particular a lo relacionado con procesos infecciosos.

Este estudio pretende determinar la incidencia de infecciones en el paciente pos-trasplante hepático, así como algunas características clínicas de su presentación, gérmenes asociados, distribución cronológica y la posible mortalidad relacionada con los procesos infecciosos. El conocimiento de estos datos permitirá realizar acciones oportunas para reducir la cantidad de este tipo de complicaciones, o reconocerlos de forma temprana para reducir la morbimortalidad asociada.

\section{MÉTODOS}

Se estudió los pacientes trasplantados hepáticos en el Hospital México y al Hospital Dr. Rafael Ángel Calderón Guardia entre el año 2000 y marzo de 2012, que presentaron o no un proceso infeccioso. Se incluyó los pacientes que cumplieran los siguientes criterios de inclusión: población con edad mayor o igual a 13 años, hospitalizada en el período comprendido entre el año 2000 y marzo de 2012, pacientes trasplantados hepáticos, sin excepción de género, etnia, país de procedencia o nacionalidad. Se excluyó los expedientes que presentaron información incompleta, de población pediátrica y con diagnóstico de infección por VIH o SIDA previo al trasplante.

No se solicitó el consentimiento informado de los pacientes incluidos por ser un estudio retrospectivo basado en la recolección de la información de los expedientes del Archivo Clínico, no haber una intervención directa sobre los pacientes y mantener la confidencialidad de la información.

Del total de pacientes de los que se obtuvo información, se comparó los sitios de infección, los síntomas más frecuentemente asociados a la presencia de un proceso infeccioso, la distribución temporal de los procesos infecciosos, los factores de riesgo para presentar un proceso infeccioso, los gérmenes causales más frecuentes de infección, los antibióticos más frecuentemente utilizados y la morbilidad y mortalidad asociadas a la presencia de una infección.

El objetivo principal fue describir la epidemiología de las infecciones que se presentan en los pacientes post-trasplante hepático y en qué lapso de tiempo suelen ocurrir dichas complicaciones.

Se condujo un estudio observacional, analítico y de cohorte retrospectivo en el que se estudió una población de 38 pacientes que presentaron un proceso infeccioso entre el año 2000 y marzo del 2012. Todos los análisis se realizaron con base en la comparación de los grupos establecidos, se tabularon las características clínicas de los pacientes, las comorbilidades, los antibióticos recibidos, los síntomas presentados, los gérmenes aislados y la letalidad asociada.

Posterior a la recolección de datos se digitó la información recopilada en una base de datos en Excel, elaborada para este estudio, a la cual una vez llenada se le realizaron pruebas de inconsistencia y errores. Los datos se procesaron en el paquete estadístico SPSS versión 20.

Se realizó frecuencias simples para todas las variables y cruces de variables para estimar la probabilidad de tener infecciones y de morir según edad, sexo y tipo de diabetes. Se creó dos bases de datos adicionales, tomando como unidad de análisis el tipo de infección y germen presentes. Los cuales se cruzaron con el año y tiempo de ocurrencia.

Los resultados de las comparaciones de variables se sometieron a pruebas de significancia estadística, para las variables cualitativas se aplicó la prueba del $\mathrm{Chi}^{2}$, bajo la Hipótesis nula de Independencia, para las variables numéricas el análisis de variancias y pruebas $t$ para valorar diferencias de promedios utilizando ANOVA, T-Test, o bien las no paramétricas como la $U$ de MannWhitney o la Prueba de Kruskal Wallis.

En los análisis se consideró significativo a un nivel de confianza $<0.05$

\section{RESULTADOS}

Las infecciones variaron entre los pacientes de 0 a 6 , siendo lo más frecuente presentar una sola infección. Nueve pacientes no presentaron infec- 
ciones, por lo que no tuvieron internamientos debido a esta causa.

De los 29 pacientes que presentaron al menos una infección, tres no fueron internados el resto, 26 de ellos, estuvieron internados entre 1 y 6 veces, siendo lo más frecuente un internamiento, en el $23.7 \%$ de los pacientes.

Tabla 1. Distribución del número de internamientos relacionados con la presencia de infección posterior al trasplante hepático por paciente.

\begin{tabular}{ccc}
\hline $\begin{array}{c}\text { Número de } \\
\text { Internamientos }\end{array}$ & Frecuencia & Porcentaje \\
\hline 0 & 3 & 7,9 \\
1 & 9 & 23,7 \\
2 & 6 & 15,8 \\
\hline 3 & 2 & 5,3 \\
\hline 4 & 6 & 15,8 \\
\hline 5 & 2 & 5,3 \\
6 & 1 & 2,6 \\
\hline Total & $\mathbf{2 9}$ & $\mathbf{7 6 . 3}$ \\
\hline Fuente: Archivo clínico Hospital México y Hospital Dr. Rafael Calderón \\
Guardia.
\end{tabular}

Gráfico No 1. Mortalidad secundaria a infección posterior a trasplante hepático.

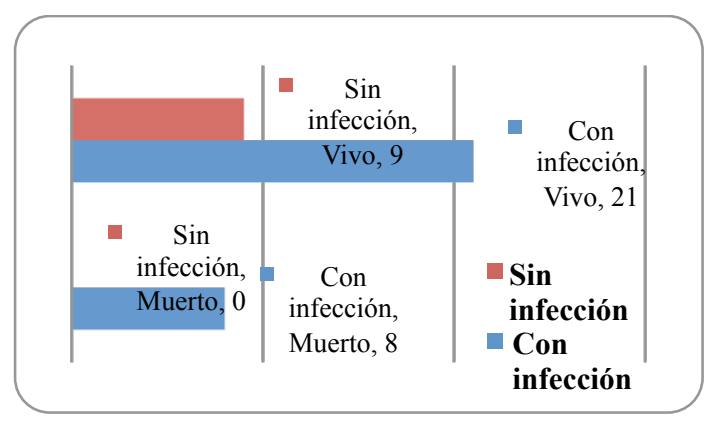

Fuente: Archivo clínico Hospital México y Hospital Dr. Rafael Calderón Guardia.

Se estudiaron 38 pacientes en los cuales ocurrió infección 29 pacientes y muerte debido a una infección el $21.1 \%$ ( 8 pacientes) para una $\mathrm{p}=$ 0.07. De los 8 pacientes que murieron, en seis de ellos la defunción ocurrió durante la primera infección, los otros dos pacientes murieron en la segunda y quinta infección respectivamente.

No hubo diferencia significativa en el riesgo de morir tomando en cuenta como factor de riesgo el antecedente de cirugía de emergencia en la población estudiada. $(\mathrm{p}=0.59)$.
Gráfico 2. Antecedente de Cirugía de trasplante hepático de emergencia.

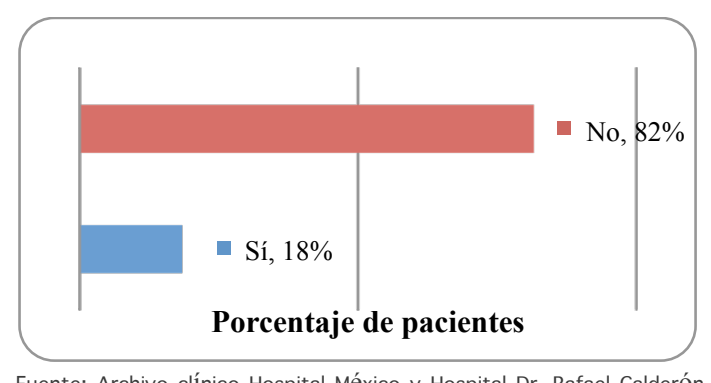

Archivo clínico Hospital México y Hospital Dr. Rafael Calderón Guardia.

En análisis de la presencia de infección y la probabilidad de morir según edad se muestra que de los 5 pacientes con edades entre 12 a 19 años, 4 de ellos $(80 \%)$ tuvieron infecciones y ninguno murió. De los 9 pacientes con edades entre 20 a 29 años ocho pacientes presentaron infección $(89 \%)$ y tres fallecieron. En los 4 pacientes en la década de los treinta todos se infectaron y uno murió. En los siete pacientes de 40 a 49 años cuatro presentaron infecciones (57\%) sin registrarse muertes. De los 13 pacientes de 50 años hasta 62 años, nueve de ellos (69\%) mostraron infecciones y cuatro fallecieron.

Además se comparó el promedio de edad entre los pacientes con y sin infecciones mostrando edades de 35.8 años y 44.8 respectivamente, valores que no resultaron estadísticamente significativos $(p=0,14)$. Tampoco mostraron diferencias significativas $(p=0.58)$ entre las edades de los que fallecieron 40.7 años y los que continuaron vivos 37.2 años.

El análisis según sexo mostró que de los 19 hombres 12 de ellos (75\%) mostraron infección y tres murieron (16\%). Entre las 22 mujeres 17 de ellas $(77 \%)$ presentaron infecciones y 5 murieron $(23 \%)$. Por lo tanto, la probabilidad de desarrollar infección $(\mathrm{p}=0.87)$ y de morir $(\mathrm{p}=0.76)$ es similar entre los géneros.

Con respecto a los diabéticos no se encontraron diferencias $(p=0.21)$ entre el valor de hemoglobina glicosilada presentada y la probabilidad de contraer infecciones, ni la probabilidad de morir $(\mathrm{p}=0.86)$. Todos los pacientes con diabetes tipo 2 desarrollaron infecciones. 
No se encontró diferencias entre la incidencia de infección $(\mathrm{p}=0.94)$ entre los pacientes que utilizaron inmunosupresión previo a trasplante hepático. Tampoco hubo diferencia en la probabilidad de morir $(\mathrm{p}=0.27)$.

Se observó una tendencia no significativa a una mayor presencia de infecciones de vías respiratorias inferiores en el primer mes posterior al trasplante, en la gran mayoría durante el mismo internamiento correspondiente a la cirugía de trasplante y por lo tanto de tipo nosocomial. En cambio la mayoría de las infecciones del tracto urinario se presentan posterior a los 7 meses del trasplante aunque dicha frecuencia de presentación tampoco tiene significancia estadística. Respecto a la detección de bacteremia, la incidencia en el tiempo es bastante uniforme en los tres cortes lineales de tiempo.

Gráfico 3. Distribución temporal de las infecciones posterior al trasplante hepático.

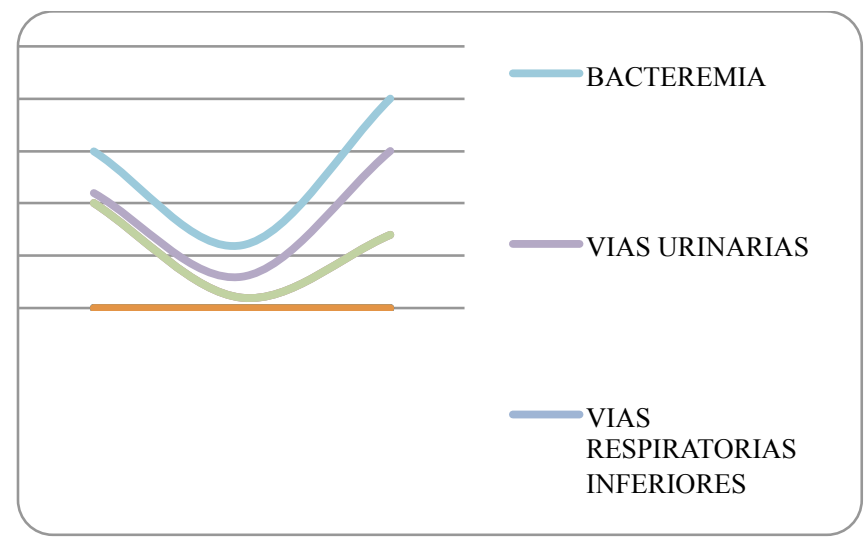

Fuente: Archivo clínico Hospital México y Hospital Dr. Rafael Calderón Guardia.

En el $66 \%$ (25 pacientes) de los 38 pacientes estudiados se aislaron gérmenes. El germen más frecuente fue Klebsiella pneumoniae metalo B lactamasas positivo el cuál se presentó en el 13\% de los pacientes.

\section{DISCUSIÓN}

Para nuestro estudio de doce años se reportaron 38 pacientes de un total de 50 elegibles que presentaron un total de 72 infecciones por diversos patógenos de origen viral, bacteriano y fúngico. De dichos pacientes sólo se logró recolectar la información de forma parcial debido a que no se encontró por parte del Archivo Clínico información correspondiente a los 12 pacientes faltantes. De estos 38 pacientes, $76 \%$ se vieron afectados por procesos infecciosos. Se observó un aumento de la incidencia acumulada en el año 2011 respecto a aislamientos de microorganismos con alta resistencia a antibióticos respecto a años previos, después del descenso evidenciado en el año 2008. Este cambio en la incidencia no fue estadísticamente significativo, más mostró una tendencia esperada tras el uso con mayor frecuencia de antibióticos de amplio espectro para tratar infecciones en los Hospitales de acopio de información.

Lo evidenciado hay que analizarlo con cautela ya que no se trasplantó el mismo número de pacientes por año; siendo el año 2011 el año que más trasplantes fueron realizados, con un número total de 15 , por lo que el incremento en la incidencia de dichos patógenos puede reflejar el sesgo de que en dicho año se trasplantaran un mayor número de pacientes.

El 76\% de compromiso de patologías infecciosas por parte de la población estudiada sigue la tendencia reportada por otros centros a nivel mundial donde se reporta una incidencia de hasta un $50 \%$ de complicaciones infecciosas posterior al primer año post trasplante ${ }^{(7)}$. Estos datos son importantes porque ya establece el precedente de cómo se ha comportado la incidencia en nuestras latitudes en la población adulta en un período de tiempo establecido.

En un estudio retrospectivo realizado en los Estados Unidos con una población de 4000 pacientes trasplantados hepáticos, seguidos durante 15 años la mortalidad reportada por causa infecciosa fue de un $28.4 \%$ de la población, cifra que incluso se encuentra discretamente por encima de los valores reportados para nuestra población en el presente estudio con un $21.1 \%$ con un seguimiento de 12 años ${ }^{(8)}$.

Respecto a la edad de defunción debido a una infección, la mayoría de los pacientes con dicho comportamiento en nuestro estudio se presentó en el grupo de etario mayor a los 50 años, dicha diferencia no se marca en una forma estadísticamente significativa en comparación a los otros grupos etarios. Un estudio realizado en España respecto a sobrevida en diversos grupos etarios posterior a trasplante hepático, de carácter retrospectivo, evidenció que de forma no significa- 
tiva, el grupo de mayor mortalidad es el que compromete a los pacientes que se encuentran entre los 40 a 50 años con un $49 \%$ de sobrevida, seguido del grupo entre los 50 a 60 años con una sobrevida del $61 \%$ y el grupo con mayor sobrevida se mantuvo entre los 30 a 40 años con $76 \%{ }^{(9)}$.

Nuestro análisis de resultados evidencia que la probabilidad de infección y mortalidad por infección tiene un comportamiento similar en ambos sexos, lo cual es concordante con el estudio realizado con pacientes españoles respecto a mortalidad, donde el género no fue un factor que evidenciará diferencias de tipo significativo respecto a mortalidad, con un $59 \%$ de sobrevida en el grupo de las mujeres versus un $57 \%$ de sobrevida en el de los hombres ${ }^{(9)}$. Por el contrario, en el estudio norteamericano previamente citado hay una marcada tendencia hacia una mayor sobrevida en el grupo femenino en todos los cortes eta$\operatorname{rios}^{(8)}$

Estudios de revisión respecto al comportamiento del paciente diabético trasplantado hepático evidencian un mayor riesgo a presentar sepsis y una mayor estadía en unidades de cuidado intensivo de forma significativa, mas dicho incremento en la morbilidad no se ve reflejado posteriormente en una mayor taza de mortalidad. En nuestro trabajo los niveles de hemoglobina glicosilada mayores a 7 no marcaron una tendencia a una mayor incidencia de infecciones, pero llama la atención que del grupo total de pacientes todos los diabéticos sufrieron una infección ${ }^{(10)}$.

Tomando en cuenta las diversas etiologías de las hepatopatías, cuyo tratamiento final fue un trasplante hepático, en nuestra serie la causa relacionada a más eventos infecciosos fue la Enfermedad de Wilson, en este grupo también hubo más muertes secundarias a infección, pero la mayoría de los pacientes trasplantados que cumplen con los criterios de inclusión para nuestro estudio, en un total de ocho; también están diagnosticados como portadores de Enfermedad de Wilson. Respecto a otros ensayos se encuentra información respecto al termino global de enfermedades metabólicas del hígado, no específicamente Enfermedad de Wilson como entidad aparte, más junto con la atresia biliar primaria y las enfermedades autoinmunes hepáticas conllevan una mejor sobrevida en comparación a otras entidades.

Después de la barrera de 5 años de sobrevida no hay diferencias significativas si se compara los grupos de pacientes trasplantados con hepatopatía terminal secundaria a hepatopatía por alcohol respecto a hepatitis autoinmune, incluso dicho comportamiento también se presenta en pacientes trasplantados por hepatitis $\mathrm{C}^{(8)}$. Los cuatro pacientes que utilizaron inmunosupresión previa al trasplante en nuestro estudio tuvieron el diagnóstico de hepatitis autoinmune.

En el período temprano pos-trasplante definido como el primer mes las infecciones nosocomiales van ser las más frecuentes, según la literatura mundial; entre ellas la neumonía asociada al ventilador, infecciones del sitio quirúrgico, y bacteremias relacionadas a catéter intravenoso. En nuestro estudio la tendencia descrita se cumple siendo las infecciones de las vías respiratorias inferiores, las más frecuentes en el primer mes posterior al trasplante hepático y dentro de este grupo se contemplaron las neumonías asociadas al ventilador, el segundo lugar en frecuencia en este lapso de tiempo lo ocupan las bacteremias cumpliendo también la tendencia descrita; y es de resaltar que de todos los episodios infecciosos sólo se documentó 1 infección en piel en el sitio quirúrgico primer mes ${ }^{(5)}$.

En la era de los años 1980 a 1990 se dio la emergencia de bacterias Gram positivas como agentes que predominantemente infectaron a los pacientes inmunosupresos incluido a los receptores de trasplante hepático ${ }^{(6)}$.

Datos emergentes sugieren que los agentes aislados tras episodios de bacteremia están cambiando en la población trasplantada. Un estudio del Grupo de Terapia Antimicrobiana Internacional (EORTC) muestra un incremento en la incidencia de gérmenes Gram negativos, tanto en la población de neutropénicos febriles como en la población post trasplante hepático, incluso excediendo a la proporción de Gram positivos aislados. Dentro de este grupo de bacterias Gram negativas cada día son más frecuentes las del grupo productor de $\beta$-lactamasas, dentro de los centros de trasplante a nivel mundial, en nuestros centros dicho comportamiento se emula siendo Klepsiella pneumoniae productora de metalobetalactamasas el germen que con más frecuencia se aisló y evidenciando una mayor incidencia a partir del años 2008, con máxima incidencia entre los años 2010 y $2011^{(11)}$.

Llama la atención que el segundo microorganis- 
mo aislado con mayor frecuencia sea del grupo fúngico propiamente la Candida albicans, retrospectivamente se documenta que en nuestros centros el fluconazol no es un medicamento que se utiliza dentro del esquema básico de profilaxis antimicrobiana lo cual podría explicar dicho comportamiento, siendo la población trasplantada una población de riesgo alto para la presencia de infecciones de tipo fúngico debido a la necesidad de uso temprano de medicamentos inmunosupresores y de antimicrobianos de alto espectro en la mayoría de los casos.

El uso de azoles como medida profiláctica ya ha sido practicado por otros centros de trasplante, mas con el tiempo del uso de dichas prácticas ha traído como consecuencia la emergencia de candidas no albicans como agentes etiológicos de candidemia hasta en un $35 \%$. Si comparamos dichos números, con nuestro medio sólo se aislaron en dos ocasiones candidas no albicans lo cual correspondería a un $25 \%$ del total, lo que está por debajo del $35 \%$ de lo descrito y como es sabido las candidas no albicans son de más difícil erradicación por el patrón de resistencia a $\operatorname{antimicrobianos}_{\text {que }} \operatorname{presentan}^{(12)}$.

De los 4 pacientes que en nuestra cohorte desarrollaron Enfermedad por Citomegalovirus documentada, tres de ellos la presentaron antes del sétimo mes, mostrando un patrón similar al descrito a la literatura donde se describe que la mayor incidencia de dicha enfermedad se da en los primeros meses, concomitante con el período de inmunosupresión. Respecto al uso de profilaxis con Ganciclovir, en nuestra población se utilizó sólo en los casos en que el receptor tuviera serologías negativas para citomegalovirus con un donador positivo, situación en la cual el riesgo de presentar una enfermedad temprana por citomegalovirus se incrementa al máximo o cuando se expresaba positividad de antigenemia en los controles postrasplante ${ }^{(13)}$.

Cabe resaltar que nuestro estudio tuvo la limitante que no en todos los casos se contó con la información que describe los antecedentes serológicos respecto a Citomegalovirus, Hepatitis $\mathrm{B}$ y Hepatitis $\mathrm{C}$, infecciones que evidentemente cumplen un papel preponderante sobre la evolución del paciente trasplantado independientemente de la etiología de la hepatopatía que llevo al paciente a presentar un trasplante hepático.

No se evaluó la variable de si el antecedente de ser un donador cadavérico o un donador vivo relacionado tuviera peso sobre la evolución del paciente posterior al trasplante; ya que sólo un paciente de la población estudiada fue trasplantado de donador vivo.

Respecto a las limitaciones del estudio, es de tipo retrospectivo por lo que sólo se pudo recopilar información correspondiente al $76 \%$ de la población elegible, a fin de obtener una muestra poblacional más significativa se homologó la población de pacientes elegibles de ambos centros a pesar que el equipo médico encargado de la realización del trasplante hepático y manejo postoperatorio es distinto, hubo sesgo por depender de encontrar el expediente en Archivo Clínico, ya sea al no encontrar el expediente, que no se anotara toda la información pertinente de parte del médico tratante o simplemente pérdida de información relevante en el expediente; algunos eventos infecciosos no fueron diagnosticados y por consiguiente no fueron tratados. Tampoco se incluyó las consultas al servicio de emergencias que no requirieron un internamiento posterior.

\section{REFERENCIAS BIBLIOGRÁFICAS}

1. O Leary JG Lepe R Davis GL. Indications for liver transplantation. Gastroenterology 2008;134:1764-1776.

2. Schiodt FV Antillasoy E Shakil AO et al. Etiology and outcome for acute liver failure in the United States. Liver Transplant Surgery 1999;5:29-34.

3. Koffron A Stein JA. Liver Transplantation: Indications, Pretransplant Evaluation, Surgery and postransplant complications. The Medical Clinics of North America 2008; 861-88.

4. O Grady JG Gimson AE O Brien CJ et al. Controlled Trials of charcoal hemoperfusion and prognostic factors in fulminant hepatic failure. Gastroenterology 1988;94: 1186-1192.

5. Shirish H. Update in Infectious Diseases in Liver Transplant Recipients. Clinics in Liver Disease 2007;337-354.

6. Fishman J Rubin R. Infection in organtransplant recipients. The New England Journald of Medicine 1998;338(24):17411751.

7. Rubin RH. The direct and indirect effects of infection in liver transplantation: Patho- 
genesis, impact, and clinical management. Curr Clin Top Infect Dis. 2002;22:125.

8. Jain A. Long-Term Survival After Liver Transplantation in 4.000 Consecutive $\mathrm{Pa}$ tients at a Single Center. Annals of Surgery. 2000;232(4):490-500.

9. Muro M. Recipient Factors Analysis in Long-Term Allograft Survival of Liver Transplantation. Transplantation Proceedings. 2002;34:290-291.

10. Preeti R. Outcome of Patients With NewOnset Diabetes Mellitus After Liver Transplantation Compared With Those Without Diabetes Mellitus. Liver Transplantation. 2002;8(8):708-713.

11. Paterson DL. Serious infections due to enteric Gram-negative bacilli-mechanisms of antibiotic resistance and implications for therapy of Gram-negative sepsis in the transplanted patient. Seminars in Respir Infect. 2002;17: 260-264.

12. Husain S Tollemar J Dominguez EA et al. Changes in the spectrum and risk factor for invasive candidiasis in liver transplant recipients: prospective, multicenter, casecontrolled study. Transplantation. 2003;75:2023-2029.

13. Van Loon AM Cleator GM Klapper PE. Human herpesviruses. In Armstrong D, Cohen D (eds): Infectious Diseases. New York, Mosby, 1999, p 8.5.1

\section{FUENTES DE APOYO}

No hay fuentes de apoyo que declarar.

\section{CONFLICTO DE INTERÉS}

No hay conflicto de intereses que declarar.

\section{AGRADECIMIENTOS}

Agradezco a la Dra. Marianella Madrigal Borloz por la orientación durante la realización de este estudio. 